DOI: $10.37393 / J A S S .2020 .01 .1$

\title{
EXERCISE UNDER DEATH-ANXIETY: INVESTIGATING INDIVIDUAL EXERCISE PSYCHOLOGY AND BEHAVIOR FROM PERSPECTIVE OF TERROR MANAGEMENT AND BEHAVIORAL IMMUNE SYSTEM INTRODUCTION
}

\author{
Xuan Pan \\ School of Sports Economics and Management, \\ Central University of Finance and Economics, Beijing, China
}

ORCID

Xuan Pan https://orcid.org/0000-0003-3554-9549

\begin{abstract}
The outbreak of COVID-19 has spread worldwide and become the world's leading health concern. Individuals are advised to stay inside and do exercise at home to avoid exposure to the disease and their psychological state is concerned. This study aims to investigate physical exercise behavior and psychological states during COVID-19, and to provide helpful suggestions for making right policies or designing effective intervention programs to help the mass respond to the pandemic.

Methods: we tested the hypotheses by conducting a national survey among 542 individuals, the data covered 28 provinces in China. Information about physical exercise habits, psychological states, perceived infection likelihood, perceived threat, attitudes towards exercise, and demographics was collected. A series of T-tests were applied to test the hypotheses.

Results: The individuals who do exercise both before and during COVID-19 outbreak increased the physical exercise frequency and reduced the categories of physical activities during COVID-19. Individuals who perceive high-level threat increased future exercise time and consumption, whereas individuals surrounded by more confirmed cases (high-level objective threat) increased current exercise frequency and intensity. With the company offamily members, individuals' exercise intention, frequency, intensity and the possibility of future exercise time and activities was significantly higher.

Conclusion: Under the threat of COVID-19, proximal defense (self-preservation) and distal defense (self-esteem enhancement) motivate individuals to increase exercise frequency. Individuals reduce types of physical activities and do exercise with family members to receive psychological security. Terror management theory and behavioral immune system provide theoretical explanations on individuals' exercise behavior and psychology.
\end{abstract}

Key words: COVID-19, terror management theory, behavioral immune system, physical exercise behavior and psychology

\section{INTRODUCTION}

The start of the 2020 s was marked by a novel coronavirus called COVID-19 (previously 2019-nCOV). This coronavirus is highly contagious, transmitted through droplets, contacts and feces, from human to human. The outbreak of the coronavirus has spread worldwide and become the world's leading health 
concern. On January 30, 2020, the World Health Organization (WHO) declared that COVID-19 outbreak is a public health emergency of international concern (PHEIC). On March 11 , WHO made the assessment on the spread and severity of COVID-19 and characterized it as a pandemic. By the end of April 16, 2020, a total of 2064,815 people had been infected and the coronavirus had killed hundreds and thousands of people (137,078 deaths) all over the world $^{1}$, causing major panic and public concerns. The number of infected people is rising in the world, posing a huge public health threat of unknown magnitude. Currently, there is no cure or vaccine available for COVID-19. Confirmed patients have to rely on their own immune system to fight against the coronavirus and get recovered. Individuals show worried, panic and anxious emotions towards COVID-19 and face death threat. To control the outbreak, the governments in some countries has taken prompt and protective measures. Italy government ordered total education shutdown to restrict the spreading of COVID-19. In China, the local government put Wuhan city (the epicenter) under quarantine, extended national holidays, closed public areas, and postponed school classes and production resumption and several provinces declared state of emergency over COVID-192.

In response to COVID-19, the public has been advised by health authorities to reduce travelling and stay at home to limit exposure to the virus (Chen, Mao, Nassis, et al., 2020). Various health authorities issued safety recommendations for taking precautions, such as wearing medical masks, washing hands, avoiding going to crowded places or mass gatherings, to reduce the exposure to the virus and its spread. For un- infected individuals, home quarantine or home stay is the fundamental safety measure to avoid infection. Although these directives are highly commendable and necessary, home quarantine during the outbreak might discourage outdoor physical activity and exercise, and increase sedentary behaviors (e.g., sitting or lying down for longer screen time, irregular sleep patterns and diets), which result in an increased risk for weight gain and physical health. For individuals with exercise habits, their physical exercise pattern would change a lot.

Moreover, Wang, Zhang, Zhao, et al, (2020) and Brooks, Webster, Smith, et al, (2020) suggest that home quarantine during COVID-19 outbreak might have negative effects on mental health. Quarantined individuals reported anxiety, depression, stress, exhaustion, detachment from others, etc. (Brooks, et al., 2020). Despite this, the ongoing pandemic situations also leads to anxiety, panic, fear and inconvenience. The worst situation is that individuals might face death threat, since COVID-19 had caused 137,078 death cases till April 16, 2020. To some extent, the outbreak discourages exercises for limited space and worries on potential infections. However, physical exercise is evidenced to be positively associated with physical and psychological health (Biddle \& Multrie, 2007; Luan, Tian, Zhang, et al., 2019). Individuals are advised to do physical exercise at home by health authorities to avoid exposure to the coronavirus and keep fit. The perceived health risk, negative psychological states, and death threat caused by COVID-19 might motivate individuals to do or increase exercise to defend against the virus. Therefore, whether COVID-19 decreases or increases individual physical exercise in reality is unclear. Furthermore, what's the relation-

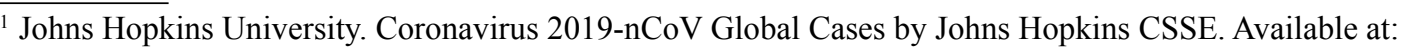
https://gisanddata.maps.arcgis.com/apps/opsdashboard/index.html\#/bda7594740fd40299423467b48e9ecf6. [accessed 16.04.2020].

${ }^{2}$ https://www.chinadaily.com.cn/a/202003/05/WS5e5ae390a31012821727b4ba.html [accessed on March 6, 2020]
} 
ship between psychological health and physical exercise behavior? In the long run, what's the effect of ephemeral psychological states formed during COVID-19 outbreak on future physical exercise and sports activities?

To answer the questions above, this study applied terror management theory (social psychology theory) and behavioral immune system (evolutionary psychology) in sports science context, and collected 542 pieces of data in a survey to explore the individual physical exercise pattern, and the relationship between physical exercise and psychological states empirically under the special condition of COVID-19 outbreak. This research is of high necessity and importance and could provide relevant public departments with guidelines for designing appropriate policies and intervention programs from sports psychology perspective, and help the mass relieve of fears and adaptation to the pandemic.

The organization of this paper is as follows. First, we discuss theoretical backgrounds of terror management theory and behavioral immune system, and how they can be applied in sports psychology context. Secondly, we formulate hypotheses on physical exercise pattern dynamics faced with pandemic, psychology of physical exercise and sports activities on the basis terror management theory and behavioral immune system. Thirdly, we test the hypotheses through a survey. Finally, this paper concludes with a discussion of research findings, theoretical and practical implications, and future research directions.

\section{THEORY AND HYPOTHESES DE- VELOPMENT}

\section{Theoretical background}

COVID-19 outbreak primed everyone's thoughts about vulnerabilities to danger and death. COVID-19 is highly contagious, for they proliferate rapidly in human's lungs once the coronavirus enters into human body, posing a life threat to individuals. Individuals who are confirmed suffer a great deal from the acute symptoms, which bring subsequent negative influence on their life, work or studies. Besides, as the number of death cases is rising and there is no available treatment or vaccine for the disease, individuals are afraid and are under death anxiety. Based on research on sports psychology, doing physical exercise is considered to be one of the ways to alleviate negative emotions, enhance self-esteem (Sonstroem \& Morgan, 1989) and enhances health conditions. The basic tenet of terror management theory suggests that individual's awareness of death and the potential overwhelming existential anxiety motivates individuals to deny mortality (Pyszczynski, Greenberg, \& Solomon, 1999), maintain faith in one's cultural world view, and acquire self-esteem in order to eliminate or reduce the existential terror (Arndt, Solomon, Kasser, \& Sheldon, 2004). Therefore, in this context, it is reasonable to apply terror management theory to address how the exercise behavior changes and explain the underlying psychology of the exercise behavioral changes during COVID-19. Besides, COVID-19 is a kind of infectious disease and research on behavioral immune system indicates that enhancing self-image is one of the ways individuals use to manage this threat of infectious disease (Ackerman, Tybur, \& Mortensen, 2018), where physical exercise could help improve self-image through maintaining a slim or strong figure. Therefore, terror management theory and behavioral immune system constitute the theoretical foundations of this research.

\section{Terror management theory}

Terror management theory suggests that like all other animals, the most basic of all human motives is an instinctive desire for continued life, or self-preservation, and the other more 
specific motives are ultimately rooted in this basic evolutionary adaptation (Pyszczynski, Greenberg, \& Solomon, 1997). The primary difference between humans and other animals is that humans possess cognitive capacities that make them explicitly and painfully aware of the inevitability of mortality. Thereby, humans have invented a meaningful defense system to manage terror. Terror management theory posits that this terror is managed by a symbolic dualcomponent cultural anxiety buffer (Pyszczynski, et al., 1997), consisting of a) an individual's personalized version of the cultural worldview, and b) self-esteem. Cultural worldview refers to beliefs about the nature of reality shared by groups of people, a set of standards through which one can attain a sense of personal value, and the promise of literal and/or symbolic immortality to those who live up to these standards (Pyszczynski, et al., 1997). Self-esteem refers to the degree to which individuals feel positive about themselves (Leary \& Baumeister, 2000), or to the sense of personal value, which is attained through believing that one is living up to the standards of value that belongs to cultural worldview. The two structures of cultural worldview and self-esteem are empirically proved to provide protection from deeply rooted fears of death and vulnerability and function to buffer anxiety resulting from mortality salience.

Furthermore, Pyszczynski et al (1999) extended terror management theory into a dual process model, which provides two general modes of proximal and distal defense. Proximal defenses refer to rational threat-focused attempts to remove conscious death-related thoughts from local attention by active suppression of such thoughts or by cognitive distortions that push the problem of death into the distant future. In contrast, distal defenses, referring to cultural worldview defense and self-esteem enhancing strategies deal with unconscious thoughts of death by making in- dividuals capable of construing themselves to be valuable in a meaningful universe. The two modes of defenses respond to the problem of death in different ways and operate in different situations (Pyszczynski et al., 1999). However, proximal defense is not a real solution to the problem of death and its effects are instant and temporal because of its limited use in coping with the ultimate fear of annihilation which emphasizes on the needs for self-esteem and faith in the cultural worldview.

Apart from that, emotional attachment proposed by Hart, Shaver and Goldenberg (2005), serves as another psychological structure to control death anxiety. Attachment theory proposes that individuals activate the attachment systems in face of threats, such as death, illness, failure and then seek support from attachment figures (e.g., parents, close friends, or romantic partners) (Bowlby, 1980; Florian, Miluincer, \& Hirschberger 2002). Rindfleisch, Burroughs and Wong (2009) found that brands or products could also be sources of emotional attachment, performing the same functions as attachment figures. Thereby, individuals could seek emotional support from familiar persons or products when they are aware of the inevitability of mortality. Combining terror management theory with attachment theory, where both theories portray human beings as vulnerable and in need of protection, support, and encouragement, the security system consisting of proximal defense, distal defense (cultural worldview, self-esteem) and attachment is constructed.

\section{Behavioral immune system}

Since individuals' biological immune systems are unlikely to have all immune-relevant genes necessary to protect the body against all types of encountered threat, in light of the costs and imperfections of biological immune systems, researchers of evolutionary psychology have hypothesized that individuals also possess 
a set of behavioral immune systems that functions as a motivational system to help protect higher organisms from bacteria, viruses, parasitic disorders and infections, avoid exposure to pathogen and mitigate the threat of disease by changing cognition, affect, and behavior before it comes into contact with the body (Schaller, 2011; Ackerman, Hill, \& Murray, 2018; Tybur \& Lieberman, 2016; Prokosch, Gassen, \& Ackerman, 2019). Behavioral immune system is seen as a complement and works as another defensive mechanism to mitigate the threat of disease before infection occurs.

Behavioral immune system exhibits functional flexibility - sensibility to the costs and benefits of pathogen avoidance, and over generality - propensity to activate even in cases where pathogen threats are absent. Functional flexibility is based on personal and contextual sensitivity, where factors implying vulnerability predict stronger responses whereas factors implying relative safety predict muted responses. Over generality reacts to the ever-changing, high-variability and hard-detection of pathogen. Although some cues like obesity, elderly appearance, disability are not infectious, behavioral immune system is activated to avoid the costs of potentially missing true disease threats.

Outputs of behavioral immune system can be classified into reactive and proactive responses (Ackerman, et al., 2018). Individuals make reactive responses in presence of information implying an immediate infection risk. These responses are generally in the form of avoidant or prophylactic behaviors (Curtis, de Barra, \& Aunger, 2011; Tybur, Lieberman, Kurzban, \& DeScioli, 2013). Proactive responses target long-term threat of illness in the form of habitual actions. For instance, daily hygiene behaviors can protect oneself from the persistent threat of bacteria and virus (Curtis \& Biran, 2001). Keeping an exercise habit to strengthen body resistance also qualify as pro- active management of long-term infection risk.

COVID-19 is highly contagious and is transmitted in various ways. Individuals worldwide are under the infection risk. Even individuals who get recovered from the coronavirus are also faced with the probability of reinfection. As health authorities suggest, avoiding exposure to the coronavirus and doing moderate exercise, both reactive and proactive, are current ways to fight against COVID-19. Therefore, behavioral immune system is appropriate for explaining the significance of doing exercise at home as a proactive response.

\section{Hypothesis development}

COVID-19 is quickly spreading all over the world, posing an urgent threat to global health. Although the fatality rate of COVID-19 is not as high as SARS and MERS, it has so far resulted in more death cases (Mahase, 2020) (COVID-19, 137,078 deaths, data updated to April 16 2020; SARS, 774 deaths; MERS: 858 deaths). The presence of COVID-19 threatens individuals' life and well-being to a great extent. Negative emotions like anxiety, worry, panic derived from COVID-19 are inevitable. From the perspective of terror management theory, the outbreak of COVID-19 motivates individuals to do physical exercise. For one reason, human being is helpless ahead of natural disasters, subject to the same need to adapt to external circumstances in order to survive and reproduce as other creatures (Solomon, Greenberg, \& Pyszczynski, 2004). During the outbreak of COVID-19, individuals stay at home surrounded by the terrible news on COVID-19 at any time. Regular physical exercise is advocated to lower perceived risk for certain diseases and health problems. When mortality concerns are conscious, health-relevant decisions (e.g. exercising) will be guided by proximal motivational goal of reducing perceived vulnerability to a health threat given the biological proclivity for self-preservation 
and removing death-related thoughts from focal attention (Ardnt, Routledge, \& Goldenberg, 2006). Bozo, Tunca and Simsek (2009) found that younger participants in temporal proximity to death show death anxiety and tend to report more health-promoting behaviors.

The psychological structure of self-esteem functions to provide protection against death anxiety. Typical ways of defending self-esteem are displaying cultural valued attributes, behaviors and achievements, fulfilling cultural valued roles, and engaging defensive reactions (Greenberg, Solomon, \& Pyszczynski, 1997). Research on psychology of sports indicates that physical exercise improves self-esteem (Sonstroem \& Morgan, 1989; Fernández-Bustos, InfantesPaniagua, et al., 2019; Tóth, Lőkös, Sipos et al., 2019), not only through enhancing self-image (shaped body figure) and then increasing selfworth, but also through conforming to the cultural values of "more exercise, more health" and the national fitness program (the State Council, 2006). Individuals who participate in a fitness program regularly or keep an exercise habit are living up to standards of values determined in the worldview. As a result, individuals could enhance their self-esteem by increasing physical exercise to avoid death anxiety caused by COVID-19. Research of Arndt, Schimel and Goldenberg (2003) demonstrates that the increase in fitness intentions offers a response to conscious death concerns (proximal defense) and functions as distal defenses through which individuals derive self-esteem and thus cope with unconscious death concerns. Therefore, it is reasonable to speculate that during the outbreak of COVID-19, individuals will increase physical exercise to cope with conscious and unconscious death concerns from the perspective of terror management theory.

Research on people's psychological responses to infectious disease also provides insights. Since no available vaccine or cure exists, the confirmed patients or uninfected persons have to rely on their own immune systems to defeat the disease. Literature on behavioral immune system suggests that exposure to pathogen cues (i.e. the novel coronavirus in this context) in external environment (health risk) would motivate avoidant or prophylactic behaviors (Tybur, Lieberman, Kurzban, et al., 2013), such as self-improvement behaviors (Ackerman, Tybur, \& Mortensen, 2018). The heightened risk of illness will lead individuals to shift energetic resources inward. In this case where individuals are threatened by the mortality risk from uncontrollable COVID-19, they are motivated to take proactive actions for survival and maintenance. Physical exercises belong to behavioral immune systems and are believed to enhance body immune system and be beneficial for one's health. Therefore, individuals would take physical exercise as preemptive behaviors to minimize the threat posed by COVID-19. Integrating research on behavioral immune system and terror management theory, we hypothesize that:

H1: During COVID-19, individuals will increase physical exercise to cope with the virus. The more death threat individuals feel, the more physical exercise they do.

In addition, the death fear caused by $\mathrm{CO}$ VID-19 not only influences the amount of individual physical exercise, but also the number of physical activity types. Ke's (2009) research found that individuals will respond to death stimuli by selecting familiar or favored brands or products, manifested as reduced types. In the same vein, individuals surrounded by the news and reports on COVID-19 will also choose familiar physical activities to maintain psychological security, which protects individuals from anxiety derived from mortality and vulnerability. From the perspective of behavioral immune system, the pathogen concerns brought by COVID-19, increases the perceived risk of uncertain decisions, leading to reduced desire 
for social affiliation with strangers or unfamiliar stimuli (Sacco, Young, \& Hugenberg, 2014; Huang, Ackerman, \& Sediovskaya, 2017). In the end, the types of physical activities individuals do during the outbreak are reduced. Taken together, we hypothesize that:

H2: During COVID-19, the types of physical activities individuals choose to do will be reduced.

As terror management theory suggests, individuals' concern on death may exert powerful effects on interpersonal affiliations and ideological allegiances. Yum, and Schenck-Hamlin (2005) found that individuals would increase interpersonal communications to eliminate or reduce terror caused by $9 / 11$ incident. When confronted with death, individuals would arouse a deeply rooted, largely unconscious desire to avoid being isolated from others (Wisman \& Koole, 2003) and respond positively to people who support their cultural values or in-group members (Pyszczynski, et al., 1997). Briefly speaking, affiliation serves as a defense of one's cultural worldviews and mortality salience increases individuals' tendency to affiliate with their in-group members (Wisman \& Koole, 2003). Besides, as the third psychological mechanism, emotional attachment suggests, individuals will activate their attachment systems and seek proximity and support from close groups instinctively when confronted with death, illness, or failure, to maintain a sense of psychological security (Hart, Shaver, \& Goldenberg, 2005). Therefore, individuals will increase contacts with close groups to acquire emotional attachments when faced with death threat.

Family members belong to the in-groups or close groups, and in most cases share the same cultural worldviews. Physical exercise is a typical way of increasing interpersonal connections and is positively associated with emotional well-being (Penedo \& Dahn, 2005; Steptoe \& Butler, 1996). Therefore, during the outbreak of
COVID-19, if one's family members also participate in physical exercise, individuals would follow family members' behaviors and do more exercise to defend cultural worldviews and relieve death anxiety. At the same time, individuals could receive emotional attachment from family members during exercises (Florian, Mikulincer, \& Hirschberger, 2002). A combined research on behavioral immune system found that disease threat was positively related to greater conformity to social norms (Ackerman, Hill, \& Murray, 2018; Murray \& Schaller, 2012; Wu \& Chang, 2012) and increased collectivism (Fincher, Thornhill, \& Murray, 2008), therefore, it is postulated that:

H3: During COVID-19 individuals will do more physical exercise in the company of family members.

Altogether, this work expands our understanding of how infectious disease COVID-19 impacts on individual physical psychology and behavior by demonstrating that COVID-19 concerns (a) motivate individuals to do more physical exercise for enhancing health conditions and self-esteem, (b) reduce types of physical activities for psychological security, and (c) induce individuals to do more exercise in the company of family members.

\section{METHODS}

The outbreak of COVID-19 across China restrained us from doing field study, therefore, online questionnaire serves as an optimum option. We conducted an online survey relying on a reputable Chinese market research institute called Wenjuanxing and examined the hypotheses. The outbreak of COVID-19 and lockdown policy of China government is a natural reminder of mortality salience. Thus, everyone is under mortality threat. Individuals are afraid of catching the virus and its subsequent terrible symptoms. Individuals' emotions and behaviors reflect real and instant reactions to death threat. 
Physical exercise is one of the ways to enhance immune systems and preserve health. To some extent, the amount and intensity of one's physical exercise reflect one's positive response to COVID-19. The questionnaire was designed to examine the influence of COVID-19 on individual psychology and exercise behaviors.

\section{Sample and Procedure}

The questionnaire was organized in the following manner: In the beginning, introductions of the survey were provided. In the informed consent form, we assured participants that the information they would provide would remain anonymous and that their participation was voluntary. In the main part, questions on daily exercise habit before and during COVID-19 were presented, including exercise motivation, perceived threat, perceived infection likelihood, perceived curability, pandemic situations, atti- tudes towards the inhibiting effects of physical exercise on COVID-19, intention to increase physical exercise time and activities. In the end, demographic information was asked.

The questionnaire was distributed in two batches. In the first batch, we distributed the questionnaire without limits throughout 28 provinces across China during COVID-19 outbreak. In the second batch, special attention was paid on areas with severe outbreaks, including Hubei, Henan, Zhejiang and Guangdong provinces. We invited participants from these four provinces to answer the questionnaire. In total, 542 participants volunteered to participate in the survey in exchange for 4-5 RMB (0.570.71 US dollars). The demographic information about the participants is provided in Table 1. No demographic differences were found between participants from the two batches. Participants' exercise information is shown in Table 2.

Table 1. Demographics of Participants (total=52)

\begin{tabular}{llccllcc}
\hline $\begin{array}{l}\text { Demo- } \\
\text { graphics }\end{array}$ & Variable & N & $\mathbf{P} \%$ & Demographics & Variable & N & P \% \\
\hline Gender & Male & 352 & 64.9 & Occupation & Migrant workers & 3 & 0.6 \\
& Female & 190 & 35.1 & (continued) & Others & 9 & 1.7 \\
Age & $<18$ & 4 & 0.7 & Education & Senior high or below & 48 & 8.8 \\
& & & & & & & \\
& $18 \sim 25$ & 134 & 24.7 & & Junior college & 80 & 14.8 \\
& $26 \sim 30$ & 158 & 29.2 & & Bachelor & 377 & 69.6 \\
& $31 \sim 40$ & 183 & 33.8 & & Master or above & 37 & 6.8 \\
& $41 \sim 50$ & 45 & 8.3 & Area & $>1000$ & 42 & 7.7 \\
& $>51$ & 31.3 & & $500-1000$ & 209 & 38.6 \\
Occupation & Students & 14.9 & & $100-499$ & 200 & 36.9 \\
& Workers & 21 & 3.9 & & $<99$ & 91 & 16.8 \\
& Medical personnel & 9 & 1.7 & Exercise history & Never do exercise & 83 & 15.3 \\
& Administrators & 191 & 35.2 & & $<1$ year & 44 & 8.1 \\
& Non-administrators & 134 & 24.7 & & $1-2$ years & 113 & 20.8 \\
& Freelancers & 39 & 7.2 & & $2-5$ years & 163 & 30.1 \\
& SECH personnel & 23 & 4.2 & & $5-8$ years & 69 & 12.7 \\
& Officials & 30 & 5.5 & & $8-10$ year & 24 & 4.4 \\
& Retired & 2 & 0.4 & & $>10$ years & 46 & 8.5 \\
\hline
\end{tabular}

Note: $N=$ number, $P=$ percentage, $S E C H=$ scientific, education, culture and health. Area is classified according to number of COVID-19 confirmed cases on 2020 February 1st in participant's current residence (province), where $>1000$ represents more than 1000 confirmed cases in the participant's current residence. For example, Hubei province has confirmed cases above 1000, so participants in Hubei province are classified as 1. Exercise history measures how long the participant keep the exercise habit, in which participants who "never do exercise" do not keep an exercise habit. 
Table 2. Exercise Frequency, Duration and Companion before and during COVID-19 outbreak

\begin{tabular}{lcccc}
\hline & Exercise before COVID-19 & Exercise & during COVID-19 \\
& $\mathrm{N}$ & $\mathrm{P} \%$ & $\mathrm{~N}$ & $\mathrm{P} \%$ \\
\hline Exercise frequency & & & 54 & 10 \\
\hline 2-3 times a day & 46 & 8.5 & 136 & 25.1 \\
once a day & 133 & 24.5 & 75 & 13.8 \\
4-5 time a week & 106 & 19.6 & 91 & 16.8 \\
2-3 times a week & 129 & 23.8 & 56 & 10.3 \\
once a week & 45 & 8.3 & 130 & 24.0 \\
Never do exercise & 83 & 15.3 & & 26.4 \\
\hline Exercise duration & & & 143 & 36.7 \\
\hline$<30$ minutes & 98 & 18.1 & 199 & 4.1 \\
30-60 minutes & 266 & 49.1 & 44 & 24.0 \\
$>60$ minutes & 66 & 12.2 & 26 & 42.8 \\
It depends & 29 & 5.3 & 130 & 27.7 \\
Never do exercise & 83 & 15.3 & & 4.8 \\
\hline Exercise companion & & & 232 & 0.7 \\
\hline Alone & 250 & 46.1 & 150 & 24.0 \\
With family member & 82 & 15.1 & 26 & 4 \\
With friends & 118 & 21.8 & 130 & \\
With club members & 9 & 1.7 & & \\
Never do exercise & 83 & 15.3 & & \\
\hline
\end{tabular}

Note: $N=$ number, $P=$ percentage

\section{Measures}

Most of our measurements were adapted from previous studies. Exercise frequency, type, duration, and intensity were used to measure individuals' weekly habitual physical exercise behavior with the questionnaire adapted from Kriska and Caspersen (1997), Godin and Shephard (1985). Exercise frequency contains six levels, $1=2-3$ times a day, $2=$ once a day, $3=4-5$ times a week, 4=2-3 times a week, 5= once a week, $6=$ never do exercises. A multiplechoice question was set to measure individuals' daily exercise type, including ball games, aerobic sports, training with fitness facilities, yoga, Kungfu, exercise with Wii or Switch, training with mobile exercise apps, etc. Exercise duration lasts from less than 30 minutes to more than 60 minutes $(1=$ less than $30 \mathrm{~min}, 2=30-60$ min, $3=$ more than $60 \mathrm{~min}, 4=$ unfixed). Exercise intensity is classified into 4 levels $(1=$ with slight body heat, $2=$ with slight body sweat, $3=$ with moderate body sweat, $4=$ with drip sweat). We also asked respondents whether there were with companions when doing exercises (ex- ercise companion). Companions range from family member, friends to club members $(1=$ alone, $2=$ with family members/relatives, $3=$ with friends, $4=$ with club members). These items constitute the composite score of individual physical exercise. The motivation to do physical exercise scales were adapted from Allender, Cowburn and Foster (2006). Individuals' emotions were measured with PANS scale (Watson, Clark, \& Tellegen, 1988), with 4-point scale where $1=$ none, $4=$ very strong. The measured emotions included anxiety, panic, anger, worry, disappointment, confusion, happiness and hopefulness.

Measures on exercise intention, perceived threat, perceived infection likelihood, perceived curability, pandemic situations, attitudes towards the inhibiting effects of physical exercise on COVID-19 were created for this study. The intention to do exercises alone or with a family member during COVID-19 was measured with a 7-point Likert scale $(1=$ do not want at all, $7=$ want to do anytime). The perceived threat of 
COVID-19 was examined through three dimensions in a 7-point scale - threat on life, threat on work $(1=$ no threat at all, $7=$ huge threat $)$, and perceived curability $(1=$ totally impossible, $7=$ totally possible). Perceived curability is a reversed item, so this item was not included when measuring reliability and validity of perceived threat construct. We asked the perceived infection likelihood of oneself, one's family member and the public in one's current residence from each participant with a 7-point scale $(1=$ totally impossible, $7=$ totally possible). Pandemic situations were calculated by summarizing whether there was a confirmed case in one's commu- nity/village, workplace/school, city/town, and whether known people got infected $(1=$ there are confirmed cases, $0=$ none). Individual's attitudes towards the inhibiting effects of physical exercise on COVID-19 (attitudes towards exercise) was measured by two items, "doing physical exercise is good for improving immune systems", "doing physical exercise helps fight against COVID-19", through 7-point scale where $1=$ strongly disagree, $7=$ strongly agree. Individuals' possibility to increase physical exercise time and sports activities in the future was examined with a 7 -point scale ( $1=$ totally impossible, $7=$ totally possible).

Table 3. Scales, Means, and Factor Loadings of Constructs

\begin{tabular}{|c|c|c|c|}
\hline Number & Items & Factor loadings & Mean (SD) \\
\hline \multicolumn{4}{|c|}{ Exercise intention during outbreak (alpha $=.859, \mathrm{CR}=.943, \mathrm{AVE}=.876$ ) } \\
\hline EID1 & Your exercise intention & .943 & $4.93(1.48)$ \\
\hline EID2 & Your family member's exercise intention & .929 & $4.56(1.49)$ \\
\hline \multicolumn{4}{|c|}{ Negative emotions $($ alpha $=.792, \mathrm{CR}=.865, \mathrm{AVE}=.615)$} \\
\hline NE1 & Anxiety & .830 & $2.53(0.76)$ \\
\hline NE2 & Worry & .790 & $2.90(0.83)$ \\
\hline NE3 & Panic & .766 & $2.35(0.89)$ \\
\hline NE4 & Disappointment & .750 & $2.32(0.87)$ \\
\hline NE5 & Anger (D) & .505 & $2.14(1.03)$ \\
\hline NE6 & Confuse (D) & .570 & $2.29(0.89)$ \\
\hline \multicolumn{4}{|c|}{ Perceived threat $($ alpha $=.695, \mathrm{CR}=.863, \mathrm{AVE}=.760)$} \\
\hline PT1 & Life & .921 & $5.13(1.47)$ \\
\hline PT2 & Work & .820 & $5.54(1.34)$ \\
\hline \multicolumn{3}{|c|}{ Perceived curability } & $5.45(1.30)$ \\
\hline \multicolumn{4}{|c|}{ Perceived infection likelihood (alpha $=.871, \mathrm{CR}=.908, \mathrm{AVE}=.767)$} \\
\hline PL1 & Yourself & .851 & $3.19(1.53)$ \\
\hline PL2 & Family member/relatives & .839 & $3.46(1.55)$ \\
\hline PL3 & The public & .935 & $3.96(1.48)$ \\
\hline \multicolumn{4}{|c|}{ Local infection situations } \\
\hline LIS1 & Whether there are confirmed case in your community/vi & age & \\
\hline LIS2 & Whether there are confirmed case in your workplace/sch & & \\
\hline LIS3 & Whether there are confirmed case in your city/town & & \\
\hline LIS4 & Whether known people get infected & & \\
\hline LIS5 & No confirmed cases around & & \\
\hline LIS6 & Not quite clear & & \\
\hline \multicolumn{4}{|c|}{ Attitudes towards exercise (alpha $=.551, \mathrm{CR}=.816, \mathrm{AVE}=.690)$} \\
\hline AE1 & $\begin{array}{l}\text { Doing physical exercise is good for improving immune } \\
\text { systems }\end{array}$ & .809 & $6.15(0.85)$ \\
\hline AE2 & Doing physical exercise helps fight against COVID-19 & .839 & $5.50(1.16)$ \\
\hline \multicolumn{4}{|c|}{ Sports activities (time and money) in the future (alpha $=.792, \mathrm{CR}=.906, \mathrm{AVE}=.827$ ) } \\
\hline $\mathrm{SC} 1$ & $\begin{array}{l}\text { Individuals' possibility to increase physical exercise } \\
\text { activities in the future }\end{array}$ & .900 & $5.22(1.36)$ \\
\hline $\mathrm{SC} 2$ & $\begin{array}{l}\text { Individuals' possibility to increase physical exercise } \\
\text { time in the future }\end{array}$ & .919 & $5.34(1.32)$ \\
\hline
\end{tabular}




\section{DATA ANALYSIS AND RESULTS}

Before examining the hypotheses, we first tested nonresponse bias and common method bias. Nonresponse bias was not a concern for our research since data were collected from an online platform. Two batches of participants volunteered and agreed to fill in the questionnaire, and no demographic differences were found between them. Given all the data were collected from a single source at one point, there was a potential for common method bias. Harman's single-factor test was applied (Podsakoff, Mackenzi, Lee et al, 2003) to check common method variance. The results of Harman's single-factor test classified the items into 12 variables with eigenvalues greater than 1, explaining a total of $79.3 \%$ variance. The first variable accounted for $25.5 \%$ of the variance, less than the benchmark $30 \%$, suggesting that common method bias was not a problem in this research.

Then, we employed confirmatory factor analysis to examine reliability and validity of the constructs. As Table 3 shows, the factor loadings of all the items were higher than 7, except two items of negative emotions which were .505 and .570 , thus we excluded the anger and confusion from the negative emotions. The values of composite reliability ranged from .816 to .943 , all greater than the threshold .7. The Cronbach's alpha coefficients of the constructs ranged from .551 to .871 , with perceived threat (alpha $=.695$ ) and attitudes towards exercise (alpha $=.551)$ lower than .7. Except attitudes towards exercise, the construct reliability was acceptable. We then examined construct validity using average variance extracted (AVE). As shown in Table 3, the values of AVE ranged from .528 to .876 , all above the benchmark .50 (Fornell $\&$ Larcker, 1981). In addition, the values of AVE were greater than square correlations of these constructs with any other variables. Thus, the convergent validity and discriminant validity is reached. Based on the above analysis, we can conclude that the measurements are reliable and valid except attitudes towards exercise.

\section{Hypothesis Testing}

As Table 2 shows, the COVID-19 outbreak discouraged individuals to do physical activities in general, as 459 vs 412 participants do exercise at least once a week before vs during the outbreak. However, Table 3 demonstrates that the average score of anxiety, worry and panic was significantly higher than 2.0 during the outbreak. Converting the variable "exercise frequency" into weekly exercise frequency, participants who did physical activities both before and during the outbreak (physical exercise activists) increased exercise frequency to a marginally significant extent $(\mathrm{N}=375)$. A paired T-test was done, and we found that they increased exercise frequency from 5.90 to 6.37 times per week marginally significantly $(\mathrm{p}<0.1$, Cohen's $d=.09)$. Besides exercise frequency, more participants did exercise alone due to home quarantine (1.73 vs $1.52, \mathrm{p}<0.00$, Cohen's $d=.23$ ). Excluding those whose exercise time was unfixed, the remaining 344 participants exercised less during COVID-19 outbreak (1.88 vs 1.78, $\mathrm{p}<0.01$, Cohen's $d=.16$ ). Therefore, we can say that they exercised more frequently, most of them did physical activities alone, but the exercises lasted less time. Their reasons for doing exercise: improving health $(\mathrm{N}=326)$, interests $(\mathrm{N}=152)$, losing weight $(\mathrm{N}=129)$ and relieving feelings $(\mathrm{N}=125)$ were the most frequently cited. The number of participants doing exercise for relieving feelings increased significantly from 99 to 125 , as shown in Table 4. 
Table 4. Frequency of motivations to do physical exercise before and during COVID-19 outbreak

\begin{tabular}{lcc}
\hline Motivations & Before COVID-19 & During COVID-19 \\
\hline Improving health & 328 & 326 \\
Interests & 188 & 152 \\
Losing weight & 169 & 129 \\
Entertainment & 54 & 49 \\
Relieving feelings & 99 & 125 \\
Killing time & 64 & 118 \\
Social interactions & 17 & 11 \\
\hline
\end{tabular}

We then analyzed the relationship between death anxiety caused by COVID-19 and individual physical exercise. Participants who did exercise at least once a week during the outbreak were examined $(\mathrm{N}=412)$. Death anxiety is measured through objective and perceived threat. We assessed objective threat ${ }^{3}$ by taking into account whether there were confirmed cases in community/village, in workplace/ school, in city/town, and whether known people got infected. If one of the four situations occurred, participants filled 1 in the blank. If two situations occurred, participants filled 2 for the same token. The larger the number, the higher the objective threat. T-test results showed that the higher the objective threat, more frequently participants did physical exercise. We divided the participants who did exercise during the outbreak into two groups according to their score in objective threat (high threat $\geq 2$; low threat $<2$ ). After comparing physical exercise of two groups, we found that the weekly exercise frequency of participants in high-threat situations was higher than that of ones in low-threat situations significantly ( 7.10 vs $5.84, \mathrm{p}<0.05$, Cohen's $d=.24$ ) and the exercise intensity was marginally higher (1.84 vs $1.70, \mathrm{p}<0.1$, Cohen's $d=.21)$. Individuals' perceived threat was measured with three items - the extent of life threat and work threat caused by COVID-19, and perceived curabil- ity. We transformed life threat and work threat into a composite variable "subjective threat" by giving equal weights. For participants who perceived huge subjective threat $(\geq 5.5)$, the possibility of increasing exercise time in the future was higher than that of the participants who perceived lower subjective threat (5.72 vs 5.26, $\mathrm{p}<0.01$, Cohen's $d=.39$ ). Moreover, for participants who perceived that COVID-19 curability was higher $(\geq 6)$, the possibility of increasing exercise time (5.61 vs 5.15, p<0.01, Cohen's $d=.38)$ and sports activities (5.74 vs 5.29, $\mathrm{p}<0.01$, Cohen's $d=.39$ ) in the future was larger. Participants who showed differences in subjective threat and perceived curability, demonstrated no significant difference in exercise frequency, intensity, and time during COVID-19 outbreak. To sum up, hypothesis 1 was supported. Physical exercise activists increased exercise frequency during COVID-19. It is interesting to find that objective threat of COVID-19 makes temporal impacts on individuals' physical exercise, such as more frequent and intense exercise, whereas perceived threat of COVID-19 makes distal impacts, such as increasing exercise time and sports activities.

Hypothesis 2 states that individuals will reduce types of physical activities in response to COVID-19. T-test showed that, for physical exercise activists, the types of physical exer-

\footnotetext{
${ }^{3}$ We use objective threat to avoid the influence of trait anxiety.
} 
cise they did before were significantly more various than that during COVID-19 (2.39 vs 2.24, $<<0.01$, Cohen's $d=.19$ ), supporting H2. Hypothesis 3 proposes that individuals will do more physical exercise in the company of family members compared with doing them alone during the outbreak. In this research, we did T-test to analyze whether participants increased exercise when accompanied by family members. The results shown in Table 5 suggest that participants increased exercise frequency and intensity during COVID-19 outbreak, and their exercise intention, the possibility of increasing future exercise time and sports activities was also higher when accompanied by family members. Compared with exercise frequency before, the gap between participants who did exercise alone and with family member was larger during COVID-19. Taken together, our results provide preliminary supportive evidence of a conceptual link between death threat caused by COVID-19 and exercise psychology and behaviors.

Table 5. T-test Results of Physical Exercise alone vs with family members

\begin{tabular}{llcccc}
\hline Period & Variables & Alone & $\begin{array}{c}\text { With family } \\
\text { members }\end{array}$ & Sig. & $\begin{array}{c}\text { Effect size } \\
\text { (Cohen's d) }\end{array}$ \\
\hline Before COVID-19 & Exercise frequency & 5.57 & 5.97 & N.s & .09 \\
(N=250 vs 82) & Exercise time & 1.91 & 1.99 & N.s & .11 \\
During COVID-19 & Exercise frequency & 5.69 & 6.69 & $\mathrm{P}<0.05$ & .21 \\
(N=232 vs 150) & Exercise time & 1.81 & 1.95 & N.s & .17 \\
& Exercise intensity & 1.59 & 1.89 & $\mathrm{P}<0.01$ & .45 \\
& Exercise intention & 5.23 & 5.59 & $\mathrm{P}<0.01$ & .33 \\
& $\begin{array}{l}\text { The possibility of increas- } \\
\text { ing future exercise time }\end{array}$ & 5.39 & 5.75 & $\mathrm{P}<0.01$ & .31 \\
& $\begin{array}{l}\text { The possibility of increas- } \\
\text { ing future sports activities }\end{array}$ & 5.30 & 5.58 & $\mathrm{P}<0.05$ & .23 \\
\hline
\end{tabular}

$\mathrm{N}=250$ vs 82 means before COVID-19 outbreak, the number of participants who did physical exercise alone was 250 , and the number of participants who did physical exercise with family member was 82 . $\mathrm{N}=232$ vs 150 means during COVID-19 outbreak, the number of participants who did physical exercise alone was 232, and the number of participants who did physical exercise with family member was 150 .

Besides the findings above, we also found response differences among different demographic groups. For all the participants $(\mathrm{N}=542)$, the possibility of increasing sports activities in the future was higher for male than female participants (Male $=5.32$ vs Female $=5.02, \mathrm{p}<0.05$, Cohen's $d=.22$ ). For indi- viduals who did exercise during the outbreak $(\mathrm{N}=412)$, male participants' exercise time was longer $($ Male $=1.95$ vs Female $=1.76, p<0.05$, Cohen's $d=.23$ ). The possibility of increasing exercise time in the future was significantly higher for female participants, compared with male participants $($ Female $=5.70$ vs Male $=5.46$, $\mathrm{p}<0.05$, Cohen's $d=.21)$. The possibility was also significantly higher for participants receiving high-level education (with bachelor's degree or above), compared with those receiving low-level education $(\mathrm{High}=5.61$ vs Low $=5.30$, $\mathrm{p}<0.05$, Cohen's $d=.26$ ). Comparing participants' attitudes towards exercise, the results indicated that participants who did exercise during COVID-19 were significantly more likely to believe in the benefits of physical ex- 
ercise in inhibiting COVID-19 than those who never did exercise $(\mathrm{Do}=5.57$ vs Never $=5.30$, $\mathrm{p}<0.05$, Cohen's $d=.23$ ), which signifies the importance of physical education.

\section{CONCLUSIONS}

\section{Discussions}

Due to the outbreak of COVID-19, individuals pay more attention to body health and realize the importance of doing exercises. This study proposes a terror management and behavioral immune system perspective to explore individuals' exercise behavioral changes and psychology during the outbreak of COVID-19 based on a national sample of 542 participants. Our results have shown that physical exercise activists increase exercise frequency to a marginal extent during the outbreak of COVID-19 and believe more in the benefits of exercise. Besides, individuals who perceive high-level threat will increase future exercise time and activities, whereas individuals with more confirmed cases around (high-level objective threat) will increase current exercise frequency and intensity. Perceived threat and objective threat impact on individuals' exercise in different ways. However, individuals from the worst hit areas (Hubei, Henan, Zhejiang and Guangdong) show no differences in exercise behavior compared with individuals from other areas. In addition, reducing the types of physical activities serves as a way to reduce anxiety as individuals could receive psychological security from familiar sports activities and reduce the risk of exploring new kinds of sports. We also found the catalytic effects of family member companion, individuals' exercise intention, frequency, intensity and the possibility of future exercise time and activities are significantly higher during COVID-19 outbreak. Affiliation with in-group members and emotional attachment from family members provide individuals with encouragement.

\section{Theoretical implications}

This research adds to a growing literature on exercise behavior and psychology, the impact of COVID-19 on cognition, and the growing body of research investigating ecological impacts on behaviors. It applies terror management theory and behavioral immune system in physical exercise behavior and psychology research, adding social psychological perspective in sports science field and providing basic understanding for exercise behavior under deadly threat.

Firstly, this research further our understanding on psychology of physical exercise, especially under a pandemic from the perspective of terror management theory and provide a novel analytical framework for sports psychology research. According to terror management theory, individuals increase exercise frequency and intensity for immediate selfpreservation or removing one's focal attention from the pandemic, as proximal defense. In addition, self-esteem and cultural worldview as distal defense motivates individuals to increase exercise, then keep an exercise habit to improve immune resistance and resolve the problem completely. Both proximal and distal defenses function to motivate individuals to do exercise, providing physical and mental protection against the death anxiety of COVID-19. We also found that the nature of death threat makes different impacts on individuals' exercise behavior, i.e. the proximity to death influences individuals' intertemporal exercise decision. As the results suggest, the higher objective threat is, the more frequently and intensely individuals do exercise here and now. The higher perceived threat is, the higher the possibility of individuals increasing future exercise time and activities. This finding is of great importance, which provides a new theoretical connection between terror management and exercise behavior. Besides, 
individuals would increase frequency, intensity, future time and activities in exercise with family members and reduce types of sports activities immediately for the need of interpersonal affiliations and emotional attachment, which are sources of psychological security. To sum up, increased exercise frequency, the companion of family member and decreased types of exercise activities can be used to cope with death anxiety induced by COVID-19. The corresponding underlying psychological mechanism - proximal defense, distal defense (self-esteem and cultural worldview), and emotional esteem as a tripartite security system serves the need of terror management.

Secondly, behavioral immune system contributes to our understanding of individuals' exercise behavior and psychology faced with coronavirus, which functions by detecting threat-relevant cues in the environment and activating responses aimed at diminishing those threats (Ackerman, et al, 2018). Doing exercise could improve health conditions and enhance self-esteem, which is a common way of diminishing threats caused by pathogen. Individuals with fit bodies will be less perceived to bear cues to infection. Therefore, the high health risk of recurrent coronavirus stimulates individuals to increase exercise as proactive actions to avoid infection. Under the threat of pathogen, this activated system also leads to cohesion with familiar and in-group targets, and aversion to unfamiliar and out-group targets, which in turn provides sufficient theoretical support for increasing exercise frequency, intensity, and intention with family member and reduced types of sports activities during the outbreak of COVID-19.

Both terror management theory and behavioral immune system provide a wealth of knowledge regarding individuals' exercise behavior and psychology during COVID-19, as illustrated above. However, terror management theory places more emphasize on individuals' reactions to death or mortality, discovering the meaning of life, or seeking symbolic longevity of humans, which guides individuals' exercise behavior and psychological recovery in a long-term. Behavioral immune system is more focused on pathogen or infection disease from the perspective of evolution, trying to investigate individuals' reactions before infection occurs, providing more specific guidelines for adaptations to COVID-19.

Thirdly, it provides an example for interdiscipline research combining sports psychology with other subjects like sociology or evolution, furthering our understanding of individual exercise behavior under extreme conditions. Future research could draw insights from other disciplines to deepen the research of sports psychology and exercise behavior. Besides, the results indicate that individuals from different groups display various exercise behavior and therefore, analysis of physical exercise behavior among different demographics groups is necessary. Demographic characteristics like gender, education need to be considered in exercise and sports psychology research.

\section{Practical implications}

This study suggests that promotion of physical exercise is beneficial in controlling COVID-19 across several dimensions. Firstly, individuals should pay attention to exercise, and then build the habit of exercising, to improve one's own physical fitness and cope with the negative emotions caused by COVID- 19 . Secondly, psychological consultants for COVID-19 could encourage patients to increase the amount of exercise, play with familiar people (family members, or close friends) or do familiar physical activities (play as usual), to manage the anxiety caused by COVID- 19 . Thirdly, the government or concerned depart- 
ments need to raise the awareness of potential physical and mental health problems of home confinement during this unusual period. Public departments related to mass sports could make some professional guidelines on physical exercise at home and provide suggestions for outdoor exercises, to alleviate individuals' psychological insecurity when exposed to big catastrophes. In the era of the Internet, everyone is connected with the world through smartphones, tablets, or computers. With the help of social media platforms (Facebook, Instagram, Sina Weibo, WeChat Moments), short video applications (Youtube, Bilibili, Tik Tok, Kuaishou), public departments could post tips of physical exercises (e.g. instructions of professionals, decomposition of actions in the form of picture or video, cautions, blogs for psychological counseling; preventive measures for outdoor exercises) to divert individuals' attention from COVID-19 and improve physical and psychological health. And last but not least, enterprises of motion sensing games, like Nintendo Switch, Wii or Xbox, could develop more kinds of indoor games to provide more choices with exercise and entertainment.

\section{Limitations and future research direc- tions}

Although this research has some interesting findings and practical implications, it is necessary to highlight the limitations and point out future research directions. Firstly, this research focuses on the direct changes of individual physical exercise behavior before and during COVID-19, and measures physical exercise, moods by scales. And because COVID-19 is highly contagious, online survey is the most appropriate way to collect data. Future research could make use of accurate methods like wearable devices to monitor individual's physical exercise and body indica- tors in a safer way. Secondly, COVID-19 is one kind of catastrophe and has its own unique influences on humans. Other kinds of catastrophes like firestorms in Australia, flu in USA, earthquakes in Japan have different impacts on individuals' physical exercise and psychological health. Individuals from different cultural backgrounds respond in mixed ways. Future research can improve our understanding of the cross-cultural impacts of big catastrophes on physical exercise. Thirdly, although the small sample size and number of comparisons made in this study limit the strength of conclusions that a reader can draw, these results provide preliminary support for the changes of individual physical exercise and psychology during COVID-19. Future research could collect longitudinal data to investigate the long-term effects of COVID-19 on individual physical exercise and psychology.

\section{Funding}

Development Fund for Young Teachers of Central University of Finance and Economics under Grant QJJ1815.

\section{REFERENCES}

Ackerman, J. M., Hill, S. E., \& Murray, D. R. (2018). The behavioral immune system: Current concerns and future directions. Social and Personality Psychology Compass, 12(2), 57-70.

Ackerman, J. M., Tybur, J. M., \& Mortensen, C. R. (2018). Infectious disease and imperfections of self-image. Psychological science, 29(2), 228-241.

Allender, S., Cowburn, G., \& Foster C. (2006). Understanding participation in sport and physical activity among children and adults: a review of qualitative studies. Health education research, 21(6), 826-835.

Arndt, J., Routledge, C., Goldenberg, J. L. (2006). Predicting proximal health responses 
to reminders of death: The influence of coping style and health optimism. Psychology \& Health, 21(5), 593-614.

Arndt, J., Schimel, J., \& Goldenberg, J. L. (2003). Death can be good for your health: Fitness intentions as a proximal and distal defense against mortality salience. Journal of Applied Social Psychology, 33, 1726-1746.

Arndt, J., Solomon, S., Kasser, T., et al (2004). The urge to splurge: A terror management account of materialism and consumer behavior. Journal of Consumer Psychology, 14(3), 198-212.

Biddle, S. J. H., Mutrie, N. (2007). Psychology of physical activity: Determinants, well-being and interventions. Routledge.

Bowlby, J. (1980). Attachment and loss: Vol. 3. Loss. New York: Basic Books.

Bozo O., Tunca A, Simsek, Y (2009). The Effect of Death Anxiety and Age on HealthPromoting Behaviors: A Terror-Management Theory Perspective. Journal of Psychology, 143(4), 377-389.

Brooks, S. K., Webster, R. K., Smith, L. E., et al (2020). The psychological impact of quarantine and how to reduce it: rapid review of the evidence. Lancet, published online Feb 26. https://doi.org/10.1016/S22150366(20)30077-8.

Chen, P. J., Mao, L.J., Nassis George, P., et al (2020). Coronavirus disease (COVID-19): The need to maintain regular physical activity while taking precautions. Journal of Sports and Health Science, 9(2), 103-104

Curtis, V., \& Biran, A. (2001). Dirt, disgust, and disease: Is hygiene in our genes? Perspectives in biology and medicine, 44(1), 17-31.

Curtis, V., de Barra, M., \& Aunger, R. (2011). Disgust as an adaptive system for disease avoidance behavior. Philosophical Transactions of the Royal Society of London B: Biological Sciences, 366(1563), 389-401.
Greenberg, J., Solomon, S., Pyszczynski,T. (1997). Terror Management Theory of SelfEsteem and Cultural Worldviews: Empirical Assessments and Conceptual Refinements. Advances in Experimental Social Psychology, 29, 61-139.

Fernández-Bustos, J. G., Infantes-Paniagua, Á., Cuevas, R., \& Contreras, O. R. (2019). Effect of Physical Activity on SelfConcept: Theoretical Model on the Mediation of Body Image and Physical Self-Concept in Adolescents. Frontiers in psychology, 10, 1537.

Fincher, C. L., Thornhill, R., Murray, D. R., \& Schaller, M. (2008). Pathogen prevalence predicts human cross-cultural variability in individualism/collectivism. Proceedings of the Royal Society B, 275(1640), 1279-1285.

Florian, V., Mikulincer, M., \& Hirschberger, G. (2002). The anxiety-buffering function of close relationships: Evidence that relationship commitment acts as a terror management mechanism. Journal of Personality and Social Psychology, 82, 527-542

Fornell, C. and Larcker, DF. (1981). Structural equation models with unobservable variables and measurement error: algebra and statistics. Journal of Marketing Research, 18(3), 382-388.

Godin, G., \& Shephard, R. J. (1985). A simple method to assess exercise behavior in the community. Canadian Journal of Applied Sport Sciences, 10, 141-146.

Huang, J. Y., Ackerman, J. M., Sedlovskaya, A. (2017). (De) contaminating product preferences: A multi-method investigation into pathogen threat's influence on used product preferences. Journal of Experimental Social Psychology, 70, 143-152.

Ke, X. (2009). Catastrophes induce less consumers' variety-seeking: a study based on the theory of terror management. Management World, 11, 122-129. 
Kriska, A. M., \& Caspersen, C. J. (1997). A collection of physical activity questionnaires for health-related research. Medicine and Science in Sports and Exercise, 29(6 Supplement), 36-38.

Leary, M. R., \& Baumeister, R. F. (2000). The nature and function of self-esteem: Sociometer theory. In Advances in experimental social psychology (Vol. 32, pp. 1-62). Academic Press.

Luan, X., Tian, X., Zhang, H., Huang, R., Li, N., Chen, P., et al (2019). Exercise as a prescription for patients with various diseases. Journal of Sport and Health Science, 8, 422-441.

Mahase, E. (2020). Coronavirus: Covid-19 has killed more people than SARS and MERS combined, despite lower case fatality rate. British Medicine Journal, 368, m641.

Murray, D. R., \& Schaller, M. (2012). Threat (s) and conformity deconstructed: Perceived threat of infectious disease and its implications for conformist attitudes and behavior. European Journal of Social Psychology, 42(2), 180-188.

Podsakoff, P. M. S. B. MacKenzie, J. Y. Lee, et al (2003). Common method biases in behavioral research: A critical review of the literature and recommended remedies. Journal of Applied Psychology, 88(5), 879-903.

Penedo, F. J., Dahn, J. R. (2005). Exercise and well-being: a review of mental and physical health benefits associated with physical activity. Current opinion in psychiatry, 18(2), 189-193.

Prokosch, M. L., Gassen, J., Ackerman, J. M., \& Hill, S. E. (2019). Caution in the time of cholera: Pathogen threats decrease risk tolerance. Evolutionary Behavioral Sciences, 13(4), 311-334.

Pyszczynski, T., Greenberg, J, \& Solomon, S. (1999). A dual-process model of defense against conscious and unconscious death-related thoughts: An extension of terror management theory. Psychological Review, 106(4), 835-845.

Pyszczynski, T., Greenberg, J., \& Solomon, S. (1997). Why do we need what we need? A terror management perspective on the roots of human social motivation. Psychological inquiry, 8(1), 1-20.

Sacco, D. F., Young, S. G., \& Hugenberg, K. (2014). Balancing competing motives: Adaptive trade-offs are necessary to satisfy disease avoidance and interpersonal affiliation goals. Personality and Social Psychology Bulletin, 40(12), 1611-1623.

Schaller, M. (2011). The behavioral immune system (and why it matters). Current Directions in Psychological Science, 20 (2), 99-103.

Solomon, S., Greenberg, J. L., \& Pyszczynski, T. A. (2004). Lethal consumption: Death-denying materialism. In T. Kasser \& A. D. Kanner (Eds.), Psychology and consumer culture: The struggle for a good life in a materialistic world (p. 127-146). American Psychological Association.

Sonstroem, R. J., \& Morgan, W. P. (1989). Exercise and self-esteem: Rationale and model. Medicine \& Science in Sports \& Exercise, 21(3), 329-337.

Steptoe, A. S., Butler, N. (1996). Sports participation and emotional wellbeing in adolescents. The Lancet, 347(9018), 17891792.

The State Council (2016). Notice of the State Council on printing and distributing the outline of the national fitness program (20162020). Issued on June 23, 2016. http://www. gov.cn/zhengce/content/2016-06/23/content_5084564.htm

Tóth, L., Lőkös, D., Sipos, K., Köteles, F., \& Szabo, A. (2019). Exercise involvement and trait-anxiety are determinants of physical self-concept: exercisers exhibit superior 
profiles compared with non-exercisers. Journal of Physical Education and Sport, 19(1), 580-585.

Tybur, J. M., \& Lieberman, D. (2016). Human pathogen avoidance adaptations. Current Opinion in Psychology, 7, 6-11.

Tybur, J. M., Lieberman, D., Kurzban, R., \& DeScioli, P. (2013). Disgust: Evolved function and structure. Psychological Review, 120, 65-84.

Wang, G. H., Zhang,Y. T., Zhao, J., et al (2020). Mitigate the effects of home confinement on children during the COVID-19 outbreak. The Lancet, published online March 04. https://doi.org/10.1016/S0140-

\section{6(20)30547-X}

Wisman, A., \& Koole, S. L. (2003). Hiding in the crowd: Can mortality salience promote affiliation with others who oppose one's worldviews? Journal of personality and social psychology, 84(3), 511-526.

Wu, B. P., \& Chang, L. (2012). The social impact of pathogen threat: How disease salience influences conformity. Personality and Individual Differences, 53(1), 50-54.

Yum, Y. O., Schenck-Hamlin, W. (2005). Reactions to $9 / 11$ as a Function of Terror Management and Perspective Taking. Journal of social psychology, 145(3), 265-286.

\section{Corresponding author:}

Xuan Pan

School of Sports Economics and Management, Central University of Finance and Economics, 39 South College Road, Haidian District, Beijing, P.R.China, 100081

E-mail: panxthu@163.com 